\title{
Are user manuals obsolete with persuasive systems?
}

\author{
Eunice Eno Yaa Frimponmaa Agyei ${ }^{1}$ and Harri Oinas-Kukkonen ${ }^{1}$ \\ ${ }^{1}$ University of Oulu, Finland
}

\begin{abstract}
Ensuring that users can successfully perform their primary tasks and achieve their behavioral goals is critical for the success of persuasive system. Are user manuals needed to achieve this goal? In this paper, we sought to understand the role of end-user documentation for persuasive systems. 50 persuasive apps from the Google Play Store were analysed to determine the importance of user manuals or guides for persuasive health apps. Although based on a small data set, our results show that in most cases persuasive systems need user documentation and hence we encourage developers of such systems to allocate resources for creating quality documentation for their users.
\end{abstract}

Keywords: User documentation, User manual, Persuasive Systems Design, Persuasive Systems, Self-explanatory user interface.

\section{Introduction}

User documentation such as user manuals, instruction sheets, quick reference guides, and troubleshooting keys are types of documentation aimed at helping users to use an information system [1]. They are used to provide information on what, when, and how to do something with a given system [2]. There exist a variety of user documentation genres including online forums, feature guides, Frequently Asked Questions (FAQ), and web-based tutorials [3]. User documentation as communication tool can enhance the value of an application to the user which in turn may improve user satisfaction [4]. Explanations provided to support the use of interactive systems have yielded benefits such as increased task completion rate [5], a reduction of computer usage anxiety [6], an increase in user satisfaction with the system [7], and better usability [8]. Despite these benefits of user documentation, often little effort, time and budget are devoted to it [9].

While the purpose of user manuals (whether in print, online, within software, on external device) is to enable people achieve their goals when using information systems, they often fail to do so. Research has shown that many end-users do not like to read user manuals [10] and this is attributed to the fact that users may often be overwhelmed by the amount of information they need to read and assimilate before using the software system [11], [12]. As a result, they skip over to sections that deals with the task they want to accomplish, skip over-explanations, prematurely ignore actions that they deem irrelevant to the tasks they want to achieve, forgo the user manual entirely and rely on from previous experience with other software systems instead of reading the provided manual. These user attitudes result in gaps in knowledge and skills required to attain the desired goal with the software [11], [12]. In addition, users are challenged with the need to 'multi-task' (i.e., read, follow the detailed instructions, and perform the tasks with the 
software). This type of multi-tasking requires the user to shift their gaze periodically from the manual to the software and vice versa. This may create a discontinuity that could lead to errors which hinders the user from achieving the intended results and may leave them perplexed about their results, and what to do next [11]. The task of developing user manuals can be daunting because the quality of the user documentation matters. Quality concerns the worth of the information content delivered to users and its aesthetics as described by [13] as well as the resources allocated to it.

The advances in the computer technology has made it possible to design apps to influence and motivate people adopt new behaviour such as exercising. Persuasive systems (PS) are "computerized software or information systems designed to reinforce, change or shape attitudes or behaviors or both without using coercion or deception" [14]. It may be easily assumed and perhaps even taken for granted that systems such as these do not need any user guides as they are so engaging per se.

In this paper, we seek to investigate the relevance of end-user documentation for persuasive systems and how they can be made feasible to support users. The paper is structured as follows. Sections 2 addresses the dual connection between end-user documentation and persuasive systems. We analyze the need for end-user documentation for persuasive systems and vice versa. Section 3 presents the study setting and results are presented in section 4. Finally, section 5 discusses the results and conclusion.

\section{Dual connectivity between end-user documentation and persuasive technologies}

End-user documentation for persuasive technologies. End-user documentation are often dismissed as irrelevant because of two popular clichés; a "well designed software needs no documentation" [15] and users do not read user manuals [16]. To counter these, firstly, it is important to note that bad software designs do exist in the real world and that people will refer to manuals if there is the need to [17]. Also, the complex and interactive features in persuasive systems necessitate the need for user manuals to improve their usability [9]. People conduct internet-based searches to obtain help with using a software. A quick google search (on 28.10.2020) produced 73,100,000 search results in 0.67 seconds for the phrase, "how to use fitbit", a leading persuasive system for physical activity self-monitoring (See Figure 1). This backs the claim that persuasive systems, among other software systems, require user manuals to support users achieve their goals. Help resources such as webpages, images and video tutorials among others were retrieved and were produced by the Fitbit company and other third-party entities such as bloggers and social media influencers. Figure 2 shows an of example user documentation available online to help users achieve their goals and the genuine need for them.

User-developed manuals differ from the vendor-developed manuals because they are more action-oriented, customized to the specific tasks, user roles, and are often shorter than those produced by the vendors according to a study by [18]. While this study was inconclusive on which of these manual types is better, it highlights the difference in user support needs and hence these findings can inform the design of end-user documentation resources. 


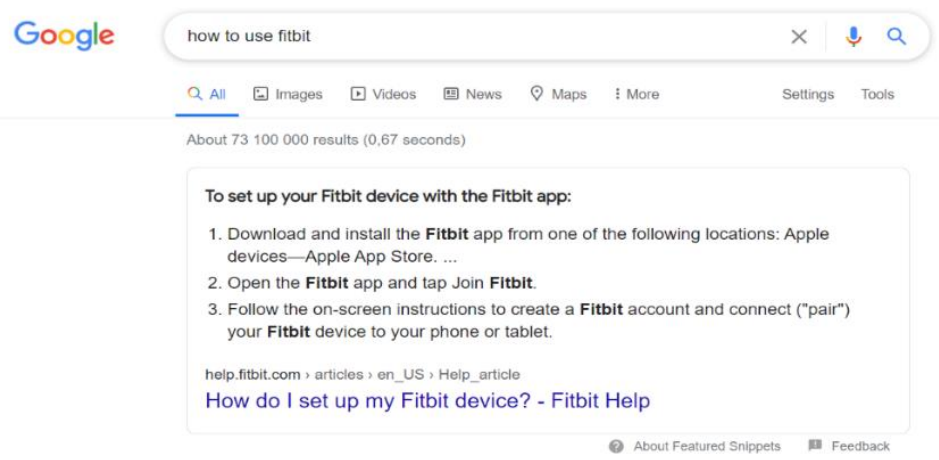

Figure 1 Example of the need for user documentation for persuasive systems.

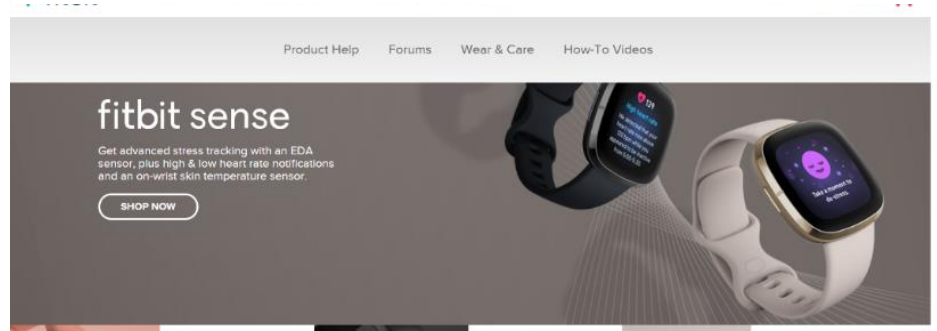

Figure 2 Fitbit's official help functionality.

Although user documentation is a useful source of support to the user, their scope may be limited due to not being dynamic or contextual enough to answer some of the questions users may have [19]. In recent years, there has been a move towards embedding instructions in the user interface also known as self-explanatory user interface (SEUI) or instructional user interface to improve user experience [5],[20][21]. Embedding instructions in the UI possess the capability to guide an end-user to interact with the system by providing information regarding the rational of the UI (e.g. the purpose of a menu item, its current state and how the state can be changed) [22] while the user is interacting with it. The goal of SEUI is to generate dynamic help systems (e.g., help message, prompts) to guide the user to perform tasks. Often these dynamic help systems are not only aware of the context of use but also the user's current task, the structure of the UI and how the UI is presented to the user [22]. They may possess the ability to reason on the application state and generate useful and valuable explanations to support the user fulfil their task.

User documentation (e.g. interactive tutorials) is used for onboarding users to an application and hence are needed for first time users to discover, learn, and engage with system functionalities, as well as to help them to achieve their goals in a timely manner [23]. This helps users to form a mental model of the application and informs their decision to use or ignore it. Also, a user documentation is an important marketing tool [24]. [25] recommends evaluating the quality of it before purchasing the software product to determine the true value of the app. It can be considered a part of the software package and its presence demonstrates professionalism and orientation of the software towards users. As a marketing tool, it can also be used to stimulate the interest of users [26]. 
Persuasive technologies for end-user documentation. End-user support documentation is key to the successful implementation and realization of the benefits of information systems [18]. Although user documentation is an auxiliary for persuasive technologies, it has to be feasible to the user in order to support the user accomplish the tasks that ultimately influence their habits, behaviours, or attitudes. To make user documentation more feasible, there is a need to determine when a persuasive system needs an end-user documentation.

\section{$3 \quad$ Research setting}

Research question. Persuasive technology includes a wide variety of technologies (e.g., web apps and mobile apps) which are available on platforms such as Android, and iOS. In this paper, we investigate and analyse persuasive apps that run on the Android platform to determine if such systems need a user manual or not. We assume here that the apps designed for a certain purpose, used by a certain group of people, produced by a company of a certain size, with certain a complexity/size of the app can mandate the need for a user documentation. The following questions guided this research:

1. Do persuasive apps published on the Google Play Store require developers to upload an end-user documentation?

2. Does the purpose/category/ user type (e.g., patients) of a PS mandate an enduser documentation?

3. Does the size of producers and resources available for a PS mandate a user documentation?

4. Does the complexity and size of a PS call for an end-user documentation?

Inclusion criteria and data extraction. Apps included in the analysis had to be published in English and available on the Android platform for free. Each app was installed on an Honor 8, an android phone that runs Android 7 operating system. Data collected included the rank of the app, total ratings, average ratings, name of the app, purpose of app, type(s) of user documentation, nature of user interface, location of the user documentation, website of the app, size of the company that produced the app, which is measured by the number of employees, the install size of the app as an indicator of the size/complexity of the app, and the category of the app (i.e., Health and fitness/Medical).

Data source. A total of 50 apps were included in the study from the health and fitness and medical categories on Play Store. We initially collected 500 health and fitness mobile apps on the 26.11.2020 from an Android app ranking website which uses the total number of app reviews as a criteria to grade apps [27]. Out of this, we selected the top 20 and bottom 15 apps to provide a broad overview of the apps within the category (most rated vs least rated). Concerning the medical category, we retrieved 15 mHealth applications listed on databases for European digital app because the apps categorized as medical by [27] were not appropriate for this review. Ten of the 15 apps were selected from the "my Health apps" repository [28] while the other five (5/10) apps from the "mHealth hub" repository [29], [30]. 


\section{$4 \quad$ Results}

Characteristics of studies. 45/50 were listed as health and fitness apps and the remaining $(5 / 50)$ as medical apps. The apps were developed for self-management of health condition (13/50), fitness tracking (10/50), weight tracking (5/50), period (i.e., menstrual cycle) tracking (4/50), sleep monitoring (3/50), drinking water tracking (2/50), medication adherence $(2 / 50)$, disease monitoring (1/50) and elderly care (1/50) purposes. The type of user documentation varied. User documentation was available for 39/50 apps which were located in the app (30/50), on the company's website (20/50), or some online resource (1/50). The companies behind these apps were classified as micro, small, medium or large using the Organization for Economic Co-operation and Development classification system for companies with a slight modification to the boundaries corresponding to less than 10 employees, between 11 and 49,50-200 and 201 or more respectively [31]. The size of company was not available for 13 out of 50 apps. The size of the apps ranged from 3.8 to 483 megabytes. The total and average ratings were available for (49/50) which indicates the perceived quality of the app [32].

When a user documentation is needed. We found that apps uploaded on the app store are required to include descriptions of the app. These descriptions tells the user what the app is for and qualify as a minimum level of user documentation (compare [1], [2]). However, the amount of information in the app descriptions varied and hence may not be enough to provide the needed support. We identified other forms of user documentation such as FAQs. All the apps evaluated had user interfaces that are self-explanatory or intuitive. Also, the apps that were listed in the medical category had user documentation(s) other than the descriptions on the Google Play store. It is important to note that apps declared 'medical' on the app store does not necessarily mean that they are 'medical apps' according to the Medical Device Regulation definitions [33] or used by patients. As such, we cannot conclude if a user documentation is needed for medical apps or not. Some apps (11/50) in the health and fitness category did not have any additional documentation. These includes four (4/11) fitness apps, two (2/11) sleep monitoring apps, two (2/11) weight tracking apps, one (1/11) activity tracking app, one (1/11) period tracking and one (1/11) self-management of health condition apps did not have any other documentation apart from the descriptions on the app store.

Different types of user manuals were produced by companies of varying sizes and available for users in the app, website, and online resources. The size of the company is associated with the amount of resources (e.g. finance and human) available and that can be allocated for creating a user documentation [34]. Our analysis shows that all the companies of small $(\mathrm{n}=5)$ and large $(\mathrm{n}=5)$ sizes produced other forms of user documentation apart from the descriptions on the Play Store. Majority of the micro-sized $(n=12 / 15)$ and medium sized $(\mathrm{n}=6 / 15)$ companies also provided other forms of user documentation in addition to descriptions on the Play Store. The ability of micro-sized companies to provide additional documentation is indeed promising if we consider the amount of resources (i.e., < 10 employees) that may be available for a user documentation.

We found that both the smallest (3.8 megabytes) and biggest app (483 megabytes) sizes had other forms of user documentation in addition to the minimum level of user documentation (i.e., descriptions on the Play Store). This finding gives an indication that 
the size of the app does not excuse the need for a user documentation. As such the availability of these end-user documentations is indeed commendable. Also, we identified that some apps $(9 / 50)$ that possessed high average ratings (i.e., $>4<5)$ did not have any other documentation apart from the minimum level of user documentation (i.e. app descriptions on Play Store) which suggests that there is no correlation between user documentation and user ratings. This finding is contrary to the research by [35] who investigated troubleshooting comments embedded in user reviews of apps listed on the Play Store. They found out that when developers responded and supported users to solve the problems users faced, user ratings of the app improved subsequently. Such troubleshooting activities reveal the need for apps to have troubleshooting content embedded in its user documentation or a separate troubleshooting user guide. This need further validates the importance of a user documentation. We believe that a user documentation that contains guides for troubleshooting is a proactive way to support multiple users to use an app instead of the passive approach via user reviews which calls for one-to-one troubleshooting activities. Providing a user documentation with the necessary information shows professionalism. In the absence of an official user manual to support users, thirdparty manuals made available by other app users, bloggers, or social media influencers can serve as an alternative source of user documentation for PSs especially those that are popular with many users (See Fitbit example in Section 2).

\section{$5 \quad$ Conclusion and discussion}

In this paper, we discussed the need and relevance of end-user documentation for persuasive systems. We analyzed 50 persuasive mobile apps. Our results give an indication that a user documentation(s) is relevant for persuasive systems. Although user documentation can be challenging to create due to limited resources, some micro and small companies identified in this study provided user documentation(s), which shows that it is possible to create a user manual regardless of the size of the company. The cliche that users do not read user manuals does not excuse the need to create one because users will consult it when they are stuck. As a PS developer, you want your users to be able to perform tasks that lead to behaviour change and not quit because they are unable to use the app due to poor-quality documentation or the lack thereof. Also, they can take advantage of the Play Store app descriptions (i.e., 80 and 4000 characters for short and full descriptions respectively) to document how their apps can be used. These descriptions can be considered as the minimum level of user documentation and together with intuitive user interfaces may provide enough support to the user. This research is a first and small step towards investigating whether user documentation is needed for persuasive systems or not. We call for more research into this topic. Future studies should investigate the impact of the type and quality of a user manual may have on task completion rates, app ratings, and perceived quality of the app. We encourage persuasive system developers to create suitable level of user documentation to support its users. 


\section{References}

[1] R. Souza and A. Oliveira, "Guide Automator: Continuous delivery of end user documentation," in Proceedings - 2017 IEEE/ACM 39th International Conference on Software Engineering: New Ideas and Emerging Results Track, ICSE-NIER 2017, 2017, pp. 31-34.

[2] G. R. Mcarthur, “If Writers Can’t Program and Programmers Can't Write, Who's Writing User Documentation?," 1986.

[3] R. H. Earle, M. A. Rosso, and K. E. Alexander, "User preferences of software documentation genres," in SIGDOC 2015 - Proceedings of the 33rd Annual International Conference on the Design of Communication, 2015, pp. 1-10.

[4] G. Torkzadeh, "The quality of user documentation: An instrument validation," J. Manag. Inf. Syst., vol. 5, no. 2, 1988.

[5] B. Myers, D. A. Weitzman, A. J. Ko, and D. H. Chau, "Answering Why and Why Not Questions in User Interfaces," 2006.

[6] B. Y. Moore, "Computer anxiety's impact on computer user documentation," 2000.

[7] L. A. Gemoets and M. A. Mahmood, "Effect of the quality of user documentation on user satisfaction with information systems," Inf. Manag., vol. 18, no. 1, pp. 47-54, Jan. 1990.

[8] N. B. Chaudhuri and D. Dhar, "Self-Explanatory Interface: Embedding Visual and Aural Syntax to Improve Usability," in Procedia Computer Science, 2020, vol. 171, pp. 18981907.

[9] D. Amalfitano, A. R. Fasolino, and P. Tramontana, "Using dynamic analysis for generating end user documentation for Web 2.0 applications," in Proceedings - 13th IEEE International Symposium on Web Systems Evolution, WSE 2011, 2011, pp. 11-20.

[10] R. Laue, "Anti-patterns in end-user documentation," in ACM International Conference Proceeding Series, 2017, vol. Part F132091, pp. 1-11.

[11] H. D. |Vanasse. S. Stolovitch, "The Paradox of User Documentation: Useful, but Rarely Used.," Perform. Instr., vol. 28, no. 7, pp. 19-22, 1989.

[12] J. Carroll and M. Rosson, "Paradox of the active user," undefined, 1987.

[13] B. K. Kahn, D. M. Strong, and R. Y. Wang, "Information Quality Benchmarks: Product and Service Performance," 2002.

[14] H. Oinas-Kukkonen and M. Harjumaa, "Persuasive systems design: Key issues, process model, and system features," Commun. Assoc. Inf. Syst., vol. 24, no. 1, pp. 485-500, 2009.

[15] B. Van Loggem, "User documentation: The Cinderella of information systems," in Advances in Intelligent Systems and Computing, 2013, vol. 206 AISC, pp. 167-177.

[16] M. Rettig, "Nobody Reads Documentation," Commun. ACM, vol. 34, no. 7, pp. 19-24, Jan. 1991.

[17] B. Van Loggem and J. Lundin, "Interaction with user documentation: A preliminary study," in ACM International Conference Proceeding Series, 2013, pp. 41-46.

[18] A. Shachak, J. Barnsley, K. Tu, A. R. Jadad, and L. Lemieux-Charles, "Understanding enduser support for health information technology: A theoretical framework," Inform. Prim. Care, vol. 19, no. 3, pp. 169-172, May 2012.

[19] A. García Frey, G. Calvary, S. Dupuy-Chessa, and N. Mandran, "Model-based selfexplanatory UIs for free, but are they valuable?," in Lecture Notes in Computer Science (including subseries Lecture Notes in Artificial Intelligence and Lecture Notes in 
Bioinformatics), 2013, vol. 8119 LNCS, no. PART 3, pp. 144-161.

[20] B. Y. Lim, A. K. Dey, and D. Avrahami, Why and Why Not Explanations Improve the Intelligibility of Context-Aware Intelligent Systems. 2009.

[21] H. C. Purchase and J. Worrill, "An empirical study of on-line help design: features and principles,” Int. J. Hum. Comput. Stud., vol. 56, no. 5, pp. 539-567, May 2002.

[22] A. G. Frey, "Self-explanatory user interfaces by model-driven engineering," in EICS'10 Proceedings of the 2010 ACM SIGCHI Symposium on Engineering Interactive Computing Systems, 2010, pp. 341-344.

[23] B. Strahm, C. M. Gray, and M. Vorvoreanu, "Generating mobile application onboarding insights through minimalist instruction," in DIS 2018 - Proceedings of the 2018 Designing Interactive Systems Conference, 2018, pp. 361-372.

[24] M. Gastegger, "Maintenance of technical and user documentation."

[25] S. Pakin, "Evaluate user documentation before you buy the software," IEEE Trans. Prof. Commun., vol. PC-24, no. 2, pp. 75-78, 1981.

[26] A. Synko and A. Peleshchyshyn, "UDC 004.91 Software development documentingdocumentation types and standards."

[27] "List of Android Most Popular Google Play Apps | androidrank.org." [Online]. Available: https://androidrank.org/android-most-popular-google-playapps?start=1\&sort=0\&price=all\&category=HEALTH_AND_FITNESS. [Accessed: 26Nov-2020].

[28] "myhealthapps.net - apps tried and tested by people like you." [Online]. Available: http://myhealthapps.net/. [Accessed: 28-Nov-2020].

[29] "European mhealth hub | Health apps repositories in Europe." [Online]. Available: https://mhealth-hub.org/health-apps-repositories-in-europe. [Accessed: 28-Nov-2020].

[30] "NHS Apps Library - NHS." [Online]. Available: https://www.nhs.uk/apps-library/. [Accessed: 28-Nov-2020].

[31] "Entrepreneurship - Enterprises by business size - OECD Data." [Online]. Available: https://data.oecd.org/entrepreneur/enterprises-by-business-size.htm. [Accessed: 27-Nov2020].

[32] E. Noei, M. D. Syer, Y. Zou, A. E. Hassan, and I. Keivanloo, "A study of the relation of mobile device attributes with the user-perceived quality of Android apps," Empir. Softw. Eng., vol. 22, no. 6, pp. 3088-3116, Dec. 2017.

[33] "Medical devices | European Medicines Agency." [Online]. Available: https://www.ema.europa.eu/en/human-regulatory/overview/medical-devices. [Accessed: 29-Nov-2020].

[34] N. C. Churchill and V. L. Lewis, "The Five Stages of Small Business Growth."

[35] S. Hassan et al., "Studying the Dialogue Between Users and Developers of Free Apps in the Google Play Store." 